\title{
Buck Chopper Simulation Based on MATLAB
}

\author{
Yanshu Niu \\ School of North China Electric Power University, Beijing 102206, China. \\ yanshu_niu@163.com
}

Keywords: Buck chopper, MATLAB, power electronics.

\begin{abstract}
Based on MATLAB software, buck circuit is simulated to deepen the understanding of buck chopper circuit principle. In this paper, the basic working principle of buck circuit is briefly described, and the component parameters of the circuit are designed. Based on the MATLAB visual simulation tool - Simulink, for several working modes of induced current, change the parameters and observe the output results. Compared with the theoretical knowledge, the research on DC / DC converter is simpler and more intuitive.
\end{abstract}

\section{Simulation Environment and Tool Box}

Simulation environment: MATLAB, Simulink

Tool-box: Start $\rightarrow$ Simulink $\rightarrow$ SimPowerSystems [1]

\section{Simulation Circuit}

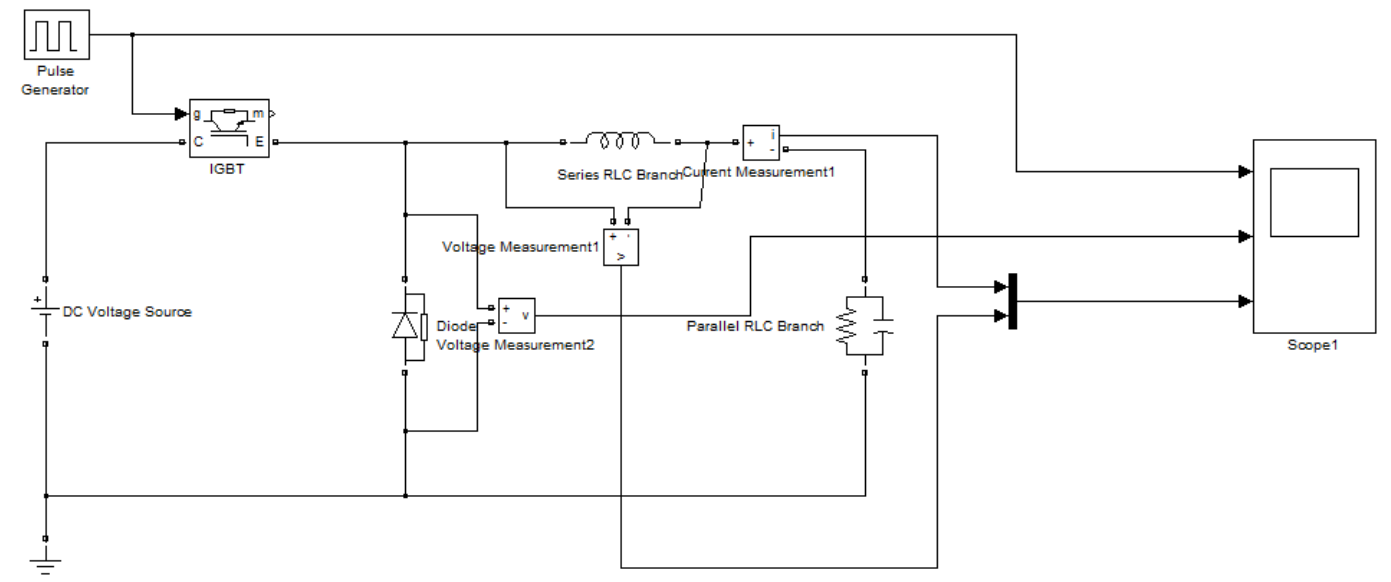

Fig 1. The simulation circuits

\section{Parameter Setting}

\subsection{Parameter Hypothesis.}
a. Input Voltage $V_{d}=200 \mathrm{~V}$;
b. Output Voltage $V_{o}=50 \mathrm{~V}$;
c. Ripple voltage is $1 \%$ of the output voltage;
d. Load resistance $R=10 \Omega$;
e. Operating frequency $f=10 \mathrm{kHz}$
According to the equation:

Duty cycle: $D=\frac{V_{o}}{V_{d}}=\frac{50}{200}=0.25$; 
Load current: $I_{o}=\frac{V_{o}}{R}=\frac{50}{10}=5 \mathrm{~A}$;

3.2 Calculation in Critical Condition.

ripple voltage:

$$
\frac{\Delta V_{o}}{V_{o}}=\frac{T_{s}^{2}(1-D)}{8 L C}=1 \%
$$

current condition:

$$
I_{L B}=I_{O B}=\frac{D T_{s}\left(V_{d}-V_{o}\right)}{2 L}
$$

According to the following equations:

Inductance: $L=0.375 \mathrm{mH}$;

Capacitance: $C=0.25 \mathrm{mF}$;

\subsection{Parameters in Simulate Circuit.}

\subsubsection{Continuous Current Working Mode.}

In order to make the inductive current continuous, the actual inductance value is 1.2 times about the threshold value [2]. So, the value of the inductance is, currently, according to the equation, the value of the capacitance is $C=0.000208 F$.

\subsubsection{Discontinuous Current Mode.}

1) is invariant

Only change the, make the value of increase, the other parameters are invariant.

2) Is invariant

Adjust decrease to make the current discontinuous.

\section{Simulation Result}

\subsection{Continuous Current Working Mode.}

When the actual inductance value is 1.2 times about the threshold value. So, the value of the inductance is $L=0.45 \mathrm{mH}$, the value of the capacitance is $C=0.000208 \mathrm{~F}$.

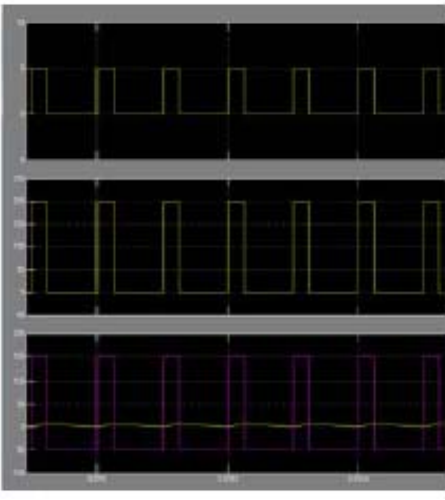

Fig 2. $D=25 \%$

\subsection{Critical Condition.}



Fig 3. $D=50 \%$

Fig $4 . \quad D=75 \%$

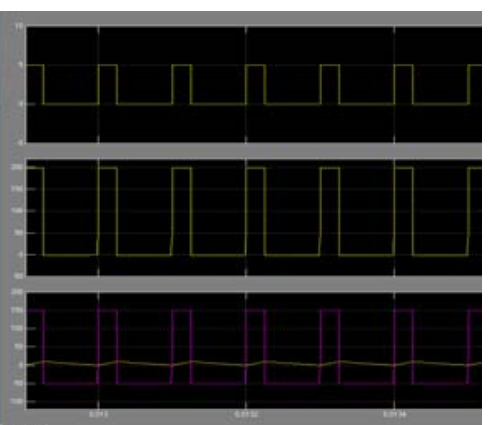

Fig 5. $D=25 \%, R=10 \Omega$ 


\subsection{Discontinuous Current Mode.}

\subsection{1 $V_{d}$ is Invariant.}

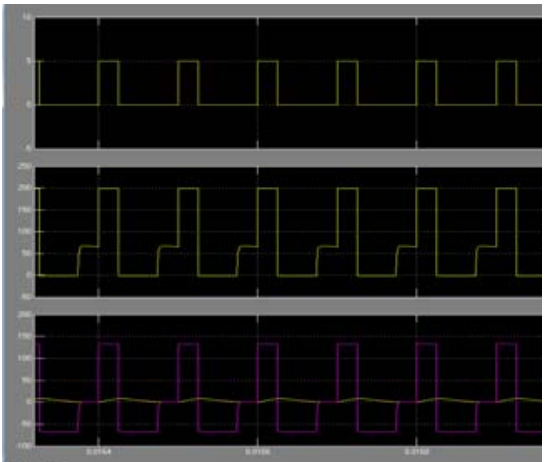

Fig 6. $V_{d}$ is invariant $D=25 \%, R=20 \Omega$

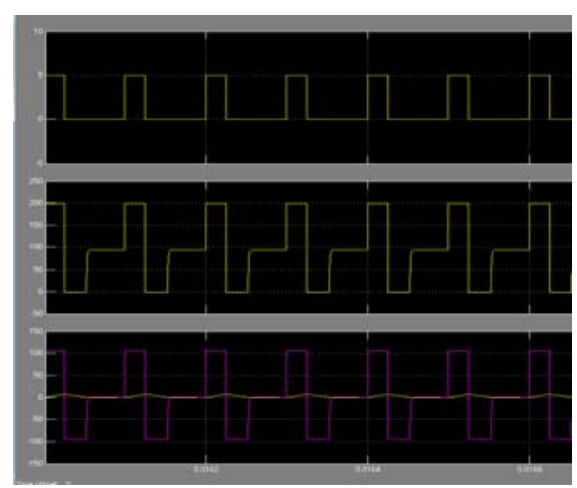

Fig7. $V_{d}$ is invariant6 $D=25 \%, R=50 \Omega$

\subsection{2 $V_{\mathrm{o}}$ is Invariant.}

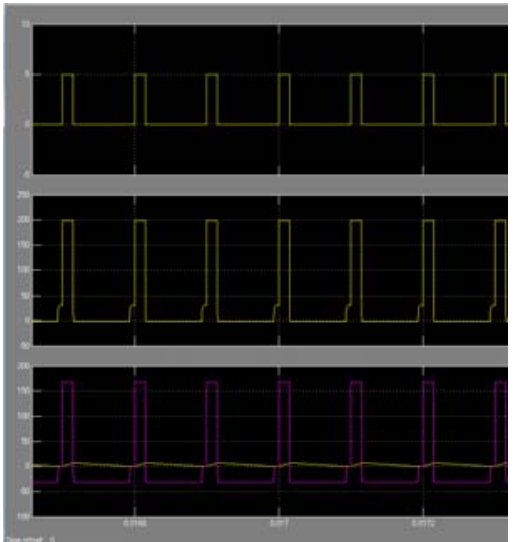

Fig $8 . V_{\mathrm{o}}$ Is invariant $D=15 \%, R=10 \Omega$

\section{Results Analysis}

The curve reflects the relationship between critical inductor average current and duty cycle: According to the following formula [3]:

$$
\begin{gathered}
I_{L B}=\frac{T_{s} V_{d}}{2 L} D(1-D) \\
I_{L B, \max }=\frac{T_{S} V_{d}}{8 L}
\end{gathered}
$$

I get the following equation:

$$
\begin{gathered}
I_{B L}=26.67 D(1-D) \\
I_{L B, \max }=6.67
\end{gathered}
$$

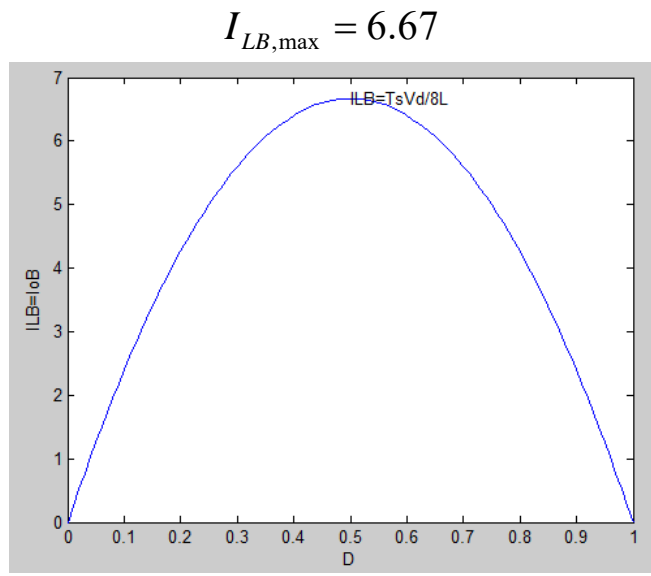

Fig 9. Relationship between average current and duty cycle of critical inductance 


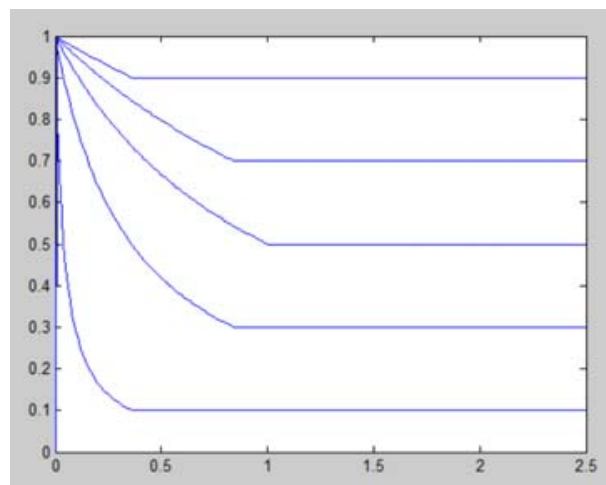

Fig 10. The critical lines for continuous and discontinuous modes

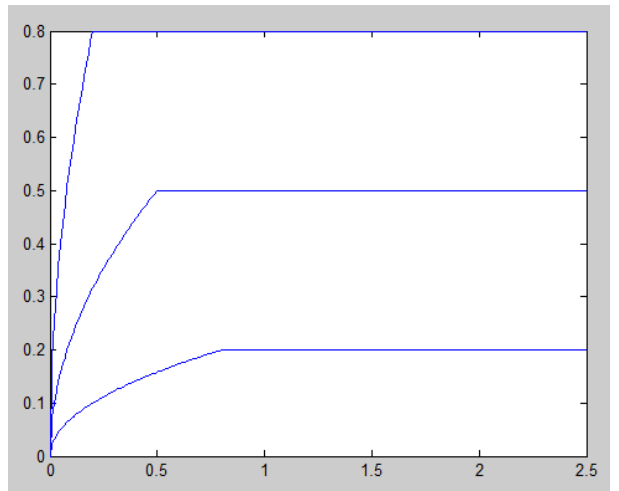

Fig 11. Characteristics curve of Buck chopper

\section{Conclusion}

a. In the case of continuous current, the output dc voltage is linearly with the switch duty cycle, and it is not related to other circuit parameters. Therefore, in the case of continuous current, the buck chopper can be regarded as the current transformer, and its equivalent ratio can be adjusted by controlling the switch duty cycle between 0 and $1[4]$.

b. In the discontinuous current mode, there are two conditions: 1) is invariant; 2) is invariant;

(1) In the case of is invariant, other parameters remain their value. When the power of output load is decreasing, in other words, the load resistance is increasing, the average inductance current will decrease, the inductance current is discontinuous.

(2) In the case of is invariant, adjust duty cycle. When the actual duty cycle is smaller than the duty cycle of critical condition, the inductance current is discontinuous.

\section{References}

[1]. Xiuqin Wang, Jun Rung, Dan Duane, Canyon He, Ting Li. Simulation of buck DC / DC converter based on MATLAB[J]. Electronic Technology,2014,43(05):5-7.

[2]. Zhang Z, Rung J, Liu X, et al. The Modelling and Simulation of DC Buck Chopper [J]. Electronic Technology, 2014.

[3]. Qigong Zhang. Principle and application of modern power electronics technology [M]. Science Press, 1999.

[4]. Chen D, Wang G. Differential Buck DC - DC Chopper Mode Inverters with High-Frequency Link [J]. Power Electronics IEEE Transactions on, 2011, 26(5):1444 - 1451. 